\title{
TITLE:
}

\section{Quantum Monte Carlo algorithm for softcore boson systems}

$\operatorname{AUTHOR}(S)$ :

makov, Jurij; Harada, Kenji; Kawashima, Naoki

\section{CITATION:}

makov, Jurij ...[et al]. Quantum Monte Carlo algorithm for softcore boson systems. Physical Review E 2003, 68(4): 046708.

ISSUE DATE:

2003-10-21

URL:

http://hdl.handle.net/2433/200795

RIGHT:

(C)2003 The American Physical Society 
PHYSICAL REVIEW E 68, 046708 (2003)

\title{
Quantum Monte Carlo algorithm for softcore boson systems
}

\author{
Jurij Šmakov, ${ }^{1, *}$ Kenji Harada, ${ }^{2, \dagger}$ and Naoki Kawashima ${ }^{3, \sharp}$ \\ ${ }^{1}$ Condensed Matter Theory, Department of Physics, Royal Institute of Technology, AlbaNova University Center, \\ SE-10691 Stockholm, Sweden \\ ${ }^{2}$ Department of Applied Analysis and Complex Dynamical Systems, Kyoto University, Kyoto 606-8501, Japan \\ ${ }^{3}$ Department of Physics, Tokyo Metropolitan University, Tokyo 192-0397, Japan
}

(Received 22 January 2003; revised manuscript received 3 June 2003; published 21 October 2003)

\begin{abstract}
An efficient quantum Monte Carlo algorithm for the simulation of bosonic systems on a lattice in a grand canonical ensemble is proposed. It is based on the mapping of bosonic models to the spin models in the limit of the infinite total spin quantum number. It is demonstrated how this limit may be taken explicitly in the algorithm, eliminating the systematic errors. The efficiency of the algorithm is examined for the noninteracting lattice boson model and compared with the stochastic series expansion method with the heat-bath-type scattering probability of the random walker.
\end{abstract}

DOI: 10.1103/PhysRevE.68.046708

PACS number(s): 02.70.Ss, 02.70.Tt, 05.30.Jp, 03.75.Hh

During the past few years there has been an increasing number of reports on strongly correlated quantum systems. A lot of attention has been focused on quantum phase transitions [1] at zero temperature, which can be observed when parameters such as the particle concentration and/or the interaction constants are varied. In order to observe the quantum phase transition experimentally, one must be able to precisely control the parameter(s), driving the transition, which is usually very difficult in real experimental situations. Therefore, only analytical theories and numerical simulations have been able to provide an accurate description of the critical behavior, associated with quantum phase transitions. Quite recently, however, a very precise tuning of parameters was achieved in a system of ultracold atoms trapped in an optical lattice, formed by the intersection of laser beams [2]. A transition from Mott insulating phase to a superfluid phase was observed. It was argued that the system is well described by the bosonic Hubbard model on a $d$-dimensional lattice, and comparisons were made with numerical simulations $[3,4]$. This is just one example of an experimental realization of a strongly correlated quantum system, and a lot of experimental work will be done along these lines in the nearest future. We believe that it is very important in such studies to be able to provide an accurate and simple theoretical description of the experimental system. Since the analytical solution of the models of strongly correlated systems is usually impossible, such a description may be in most cases provided only by the numerical simulations.

While efficient and powerful quantum Monte Carlo (QMC) algorithms exist for general quantum spin systems, the progress in the development of the algorithms for the numerical simulations of bosonic systems with no hard core is much more modest. In the present paper we describe a QMC algorithm, allowing efficient simulations of the bosonic models with short-range interactions on a lattice in

\footnotetext{
*Electronic address: jurijus@condmat.physics.kth.se

†Electronic address: harada@acs.i.kyoto-u.ac.jp

‡Electronic address: nao@ phys.metro-u.ac.jp
}

the grand canonical ensemble, with average particle number controlled by the chemical potential.

Recently an efficient QMC algorithm for the simulation of spin models with arbitrary spin quantum number $S$ on the lattice was proposed and implemented [5]. It is based on coarse graining of the conventional loop algorithm with split-spin representation, in which each spin- $S$ operator is replaced by a sum of $2 S$ Pauli matrices. One update cycle of worldline configuration in this algorithm consists of (a) placement of the vertices on the space-time lattice; (b) creation of a pair of spin-raising or spin-lowering worms; (c) propagation of one of the worms through the lattice with scattering on the vertices, resulting in changes of worldline configuration; (d) worm annihilation. The algorithm for a particular model is thus defined by specifying a number of parameters, depending on the local worldline configuration: density of vertices, scattering probabilities at vertices, and the probabilities for creation and annihilation of a pair of worms.

Holstein-Primakoff (HP) transformation [6] gives a relation between the spin systems and the boson systems. In spin wave theories, the transformation is used for mapping a spin problem into a boson problem. Here we do the opposite in order to derive a Monte Carlo algorithm for bosonic systems from the above-mentioned one for spin systems. The relation can be written as $S_{i}^{+}=b_{i}^{\dagger}\left(2 S-b_{i}^{\dagger} b_{i}\right)^{1 / 2}, \quad S_{i}^{-}=(2 S$ $\left.-b_{i}^{\dagger} b_{i}\right)^{1 / 2} b_{i}$, and $S_{i}^{z}=n_{i}-S$, where $S_{i}^{+}, S_{i}^{-}$, and $S_{i}^{z}$ are spin operators on the site $i$, and $b_{i}^{\dagger}$ and $b_{i}$ are the boson creation and annihilation operators. At a first glance it appears that the algorithm derived from the HP transformation would be directly applicable only to the boson systems that have an artificial limitation of number of particles per site (i.e., it cannot exceed $2 S$ ). We show that this is not the case in the following.

We lift the limitation by taking the limit of large $S$. Examining the HP transformation, we note that if $n_{i}$ will be kept finite by the chemical potential, in the large $S$ limit we can neglect higher order terms making no error, and keep only the lowest order in $n_{i}=b_{i}^{\dagger} b_{i}$ in the HP transformation which leaves us with 


$$
b=\frac{1}{\sqrt{2 S}} S^{-} \quad \text { and } \quad b^{\dagger}=\frac{1}{\sqrt{2 S}} S^{+} .
$$

Mapping (1) allows us to rewrite the Hamiltonian of a bosonic model in terms of spin operators. Thus, if there is an algorithm for spin systems with arbitrary $S$, and if the infinite $S$ limit of this algorithm exists, we can easily obtain an algorithm for the bosonic systems.

To demonstrate this idea, we consider a simple model of noninteracting softcore bosons on a $d$-dimensional hypercubic lattice of linear size $L$ with the Hamiltonian

$$
H=-\frac{t}{2} \sum_{\langle i j\rangle}\left(b_{i}^{\dagger} b_{j}+b_{j}^{\dagger} b_{i}\right)-\mu \sum_{i} b_{i}^{\dagger} b_{i},
$$

where $t$ is the (positive) hopping amplitude, $\mu$ is the chemical potential, and the first sum is over the pairs of nearestneighbor sites. Using mapping (1) we can replace the bosonic operators in Eq. (2) with the spin operators, leading to a model equivalent to the original bosonic model in the limit of infinite $S$ :

$$
H=-\frac{t}{4 S} \sum_{\langle i j\rangle}\left(S_{i}^{+} S_{j}^{-}+S_{j}^{+} S_{i}^{-}\right)-\mu \sum_{i} S_{i}^{z} .
$$

Since this is an $X Y$ spin model, an efficient algorithm is available for any $S$ [5]. Our task is, therefore, to take the infinite $S$ limit of the algorithm. It turns out that all the parameters defining the coarse-grained algorithm have welldefined values in this limit as well. Below we describe the procedure of taking this limit and give a detailed description of the softcore boson algorithm for the noninteracting model. Generalizations to models with interactions, such as the onsite repulsive interaction and short-ranged repulsive and/or attractive interactions, are straightforward. This, for instance, makes the present idea readily applicable to the boson Hubbard model.

Naturally, boson occupation number must be positive, which leads to a restriction on the possible values of chemical potential: $\mu<-d t$ or $|\mu|>d t$. To apply the coarsegrained algorithm we can use the values of parameters, derived for the general $X X Z$ model in Table I of Ref. [5]. Relationship between the parameters in Ref. [5] and the parameters of our model is

$$
h=-\frac{|\mu|}{2 d S}, \quad J=\frac{t}{2 S}, \quad J^{\prime}=0 .
$$

One has to use the results of Ref. [5] with caution, since they are given for the case of positive $h$. Therefore, in order to use them for the present problem, we need to change the sign of the field in Eq. (4) and at the same time reinterpret particle numbers denoted by $l$ and $m$ in Ref. [5]. Namely, in the present paper $l$ denotes the number of holes, whereas $\bar{l}$ $\equiv 2 S-l$ denotes the number of particles. Accordingly, while taking the infinite $S$ limit with fixed density of particles we have to assume that $l$ and $m$ are close to $2 S$, whereas $\bar{l}$ and $\bar{m}$ are of the order of unity.
Probability of creation of a pair of spin-raising or particlenumber-decreasing (PND) worms in the coarse-grained algorithm is $\bar{l} / 2 S$ and that of a pair of lowering or particlenumber-increasing (PNI) ones is $l / 2 S$. By taking the limit $S \rightarrow \infty$ we find that the probability to create a pair of PND worms is zero. Corresponding probability for a pair of PNI worms is then unity, indicating that our cycle will always start with a pair of PNI worms. That, however, does not mean that the number of particles will be constantly increasing, since the worm changes its type to the opposite one every time it changes direction as a result of scattering on a vertex. Once the traveling worm returns to the point of origin, it can either annihilate there, ending the cycle, or pass through. The probability of annihilation of a pair of PND worms is $1 / \bar{l}$ and zero for the PNI ones.

Remaining parameters, such as density of vertices and the vertex scattering probabilities, needed for the construction of the algorithm, can be derived by examining the values in Table I of Ref. [5] for region IV and taking the value of $S$ to infinity. First of all, the vertex density $B$ is given by $B$ $=h(l m+l \bar{m}+\bar{l} m) / 2$. To list nonzero scattering probabilities, using the notation of Ref. [5], we have

$$
\begin{gathered}
P\left(\downarrow \mid \begin{array}{cc}
l & m \\
l^{-} & m
\end{array}\right)=2 S(h-J) /(2 B), \\
P\left(\nearrow \mid \begin{array}{cc}
l & m \\
l^{-} & m
\end{array}\right)=m J /(2 B), \\
P\left(\rightarrow \mid \begin{array}{cc}
l & m \\
l^{-} & m
\end{array}\right)=\bar{m} J /(2 B), \\
P\left(\nearrow \mid \begin{array}{cc}
l+1 & m \\
l^{+} & m+1
\end{array}\right)=1 / \bar{l}, \\
P\left(\rightarrow \mid \begin{array}{cc}
l-1 & m \\
l^{-} & m-1
\end{array}\right)=1 / l .
\end{gathered}
$$

Here we have set $J^{\prime}=0$ and the superscript + or - indicates that the type of the incoming worm is PND or PNI, respectively. Seemingly, there is a problem with density of vertices becoming infinite in the infinite $S$ limit. However, it should be noted that all nontrivial probabilities of the scattering events are proportional to $1 / B$, so that the density of the scattering events remains finite. In other words, in the limit of infinite $S$ the situation is identical to the one that occurs when taking the continuous imaginary time limit in a conventional loop algorithm [7]. Exploiting the analogy to the continuous imaginary time loop algorithm, we can easily construct a procedure for finding the time of the next scattering event. Namely, instead of examining each vertex, it is possible to generate the time of next event as a Poissondistributed random number where the average time interval or the density depends on the local spin configuration and the type of the scattering process.

We can readily obtain the density of such events by multiplying the scattering probabilities $(5)-(7)$ by $B$, and take 
the infinite $S$ limit. Since Eq. (7) yields zero, we have two nonzero scattering densities for intervals that have no kinks in it:

$$
\Lambda\left(\downarrow \mid \begin{array}{cc}
l & m \\
l^{-} & m
\end{array}\right)=\frac{|\mu|-d t}{2 d}
$$

and

$$
\Lambda\left(\nearrow \mid \begin{array}{cc}
l & m \\
l^{-} & m
\end{array}\right)=\frac{t}{2}
$$

For the scattering probability at kinks, only the scattering probability (8) will remain nonzero, since probability (9) vanishes in the infinite $S$ limit.

In order to describe the algorithm in detail, we introduce a concept of a constant environment interval (CEI) on which the moving worm resides. A CEI is defined as an interval ahead of the worm in which the environment of the worm does not change in the imaginary time direction, i.e., the worldline state changes neither on the current site nor on any of the neighboring sites. This interval is bounded by one of three events, closest to the worm: (a) a kink on the current site, (b) a kink on one of the neighboring sites, or (c) the point of origin, where the other worm waits for the moving worm.

Worldline configuration update cycle for the noninteracting model may be summarized as follows.

(1) Choose an arbitrary space-time point to place a pair of worms, one of which will move, producing the changes in the configuration, and another one will mark the point of origin. Always start with a PNI (spin-lowering) worm. Choose the arbitrary direction (up or down) for the worm's initial movement.

(2) Determine the CEI.

(3) For each type of scattering and for each nearestneighbor site, which is a candidate for the final scattering destination, generate the time of the next possible scattering event stochastically according to the Poisson distribution with densities (10) and (11).

(4) If the advancement of the worm by the smallest of these times does not take the worm out of CEI, implement the corresponding scattering event. In case of a backscattering event, change the type of the worm to the opposite one. Go back to (2).

$\left(4^{\prime}\right)$ If the advancement of the worm gets the worm out of CEI, advance the worm to the end of the CEI.

(5) If the end point of the CEI is not a kink or the original starting point, go back to (2).

$\left(5^{\prime}\right)$ If the end point of the CEI is a kink, attempt to scatter on it according to Eq. (8). If the scattering fails or not applicable, let the worm skip the kink and go on. Go back to (2).

$\left(5^{\prime \prime}\right)$ If the end point of the CEI is the original starting point, stochastically determine, whether they will annihilate with the probability $1 / \bar{l}$ where $\bar{l}$ is the particle number on the CEI. If it annihilates, the update cycle is terminated. Otherwise go back to (2).

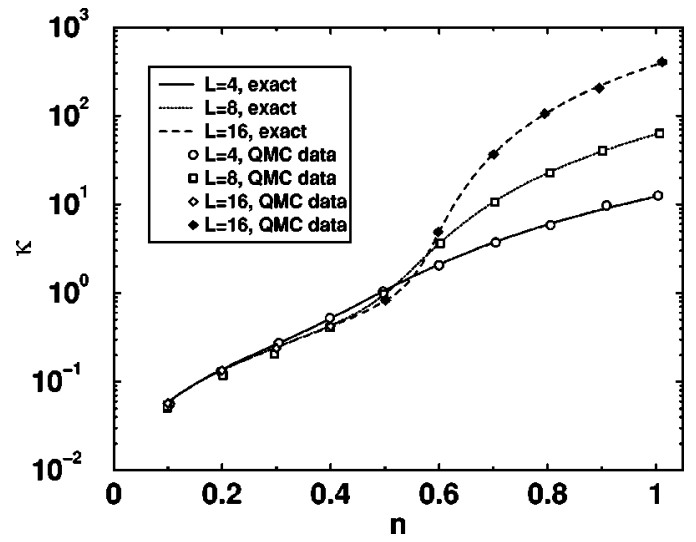

FIG. 1. The compressibility plotted against the average occupation number for three-dimensional free lattice boson system at $k_{B} T=2.0$. The lines are the exact analytical values, while the symbols are the results of QMC simulation. Uncertainty in the occupation number is in all cases smaller than the width of the symbols. Data for $L=16$ and $\langle n\rangle>0.4$ are computed with the standard number of cycles (solid diamonds), while the data for $\langle n\rangle \leqslant 0.4$ were obtained with a 100 times longer simulation (open diamonds). Error bars are in all cases smaller than the size of the symbols.

After a number of full update cycles (resulting in worm annihilation) the observables are measured.

To test the validity and evaluate the efficiency of the algorithm we have performed a number of tests, comparing the results of QMC simulation of the noninteracting boson model in three dimensions to the exact results. Although the model does not have any interaction terms, it is nontrivial enough to provide us with excellent grounds for testing because it displays Bose-Einstein condensation (BEC) and the observables may be calculated analytically.

We have performed simulations at $k_{B} T=2$ (here and below we use $t$ as the unit of energy, by putting $t=1$ ) at ten different values of the chemical potential, chosen so that the resulting average occupation number would be $n \equiv\langle N\rangle / V$ $=0.1,0.2, \ldots, 1.0$. Three different system sizes are considered: $L=4,8,16$. If not stated otherwise, for each value of system size and chemical potential we have performed 50000 cycles for equilibration, and another 50000 cycles for measurement. The 50000 measurement cycles were divided into ten bins of the equal length for estimating the statistical error.

In all cases we investigated, including cases close to criticality and ones deep inside the superfluid phase, we found an excellent agreement between the numerical QMC data and the exact analytical results. As an example, Fig. 1 shows the dependence of the compressibility $\kappa \equiv(\partial n / \partial \mu)_{T}$ $=\left(k_{B} T\right)^{-1}\left(\left\langle N^{2}\right\rangle-\langle N\rangle^{2}\right) / L^{d}$ as a function of $n$ at a fixed temperature $k_{B} T=2$. The observation of the divergent behavior of compressibility at BEC transition is well within the reach of numerical simulation. For low values of $n$ and $L$ $=16$ we had to increase the number of cycles 100 times in order to obtain a good statistics because the typical lifetime of a worm becomes too short in this case. Even after this increase, the CPU time spent for this case is smaller than that for the superfluid cases.

In Fig. 2, we plot the superfluid density $\rho_{S}$ against the 


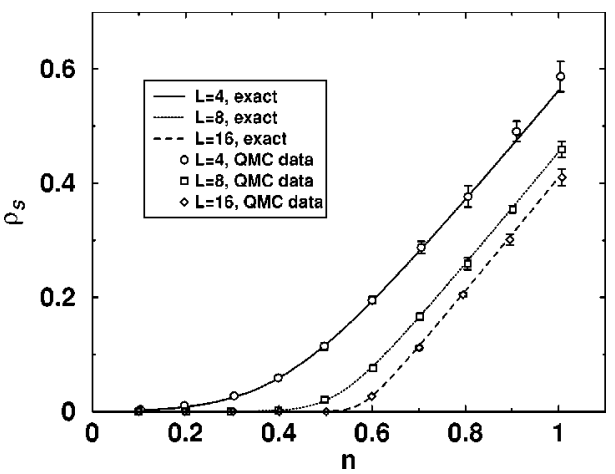

FIG. 2. The superfluid density plotted against the average occupation number for three-dimensional free lattice boson system. Standard simulation parameters were used for all data points. The lines are the exact analytical values while the symbols are the results of QMC simulation.

average occupation number. $\rho_{S}$ is defined [8] as $\rho_{S}$ $\equiv L^{-d} k_{B} T\left[d^{2} F(\theta) / d \theta^{2}\right]_{\theta=0}$ where $F(\theta)$ is the free energy of a system twisted by the angle $\theta$ per lattice spacing. In QMC simulation this quantity can be measured by $\rho_{\mathrm{S}}$ $=L^{2-d} k_{B} T\left\langle W_{x}^{2}\right\rangle$, where $W_{x}$ is the sum of winding numbers of all worldlines in the $x$ direction. The possibility of measuring the winding number fluctuation is one of the advantages of the present approach, compared to the algorithms, such as the one used in Ref. [4], which works in the fixed winding number ensemble. In Fig. 2, we can again see that the onset of the condensation is captured by the QMC simulation with the present algorithm.

We have also compared the performance of the present algorithm with the directed loop algorithm [9], one of the best QMC algorithms currently available for the simulation of softcore boson systems. The directed loop algorithm is quite general and powerful method, applicable, in principle, to any quantum system. However, it is up to the user to find a set of scattering probabilities, optimizing the efficiency of the algorithm for a given model. Due to a huge freedom in the choice of algorithm parameters, in most cases this is a highly nontrivial task. Also, to apply the directed loop algorithm to the softcore boson systems, one has to set an artificial upper bound for the site occupation number. This upper bound must be taken large enough to make the simulation free from the systematic error. For comparison purposes we have used the directed loop algorithm with a set of simple heat-bath scattering probabilities and the site occupation number was limited by $n_{i} \leqslant 20$.

To perform a quantitative comparison of algorithm performance, we have constructed an estimator for the integrated autocorrelation time (IACT) by measuring bin averages of observables for different bin sizes. Denoting the variance of a set of averages over the bins of size $m$ by $V_{m}$, the IACT $\tau$ may be estimated as a large $m$ limit of

$$
\tau(m)=\frac{m V_{m}}{2 V_{1}},
$$

where $V_{1}$ is the variance of the individual measurements. This allows us to determine the IACT by plotting $\tau(m)$ as a
TABLE I. The integrated autocorrelation time (IACT) for the occupation number and the superfluid density, measured for threedimensional systems with linear sizes $L=4,8$ at different values of the average occupation number $\langle n\rangle$ using the bin averaging technique (see text). The IACT is expressed in terms of the average number of scattering events, experienced by the worm. Error bars are estimated from four runs with identical parameters and different random number generator seeds.

\begin{tabular}{lccc}
\hline \hline$L$ & $\langle n\rangle$ & Proposed algorithm & \multicolumn{2}{c}{ SSE heat-bath algorithm } \\
\hline \multicolumn{3}{c}{ Occupation number IACT } \\
4 & 0.2 & $265 \pm 50$ & $(28.3 \pm 3.2) \times 10^{4}$ \\
4 & 0.4 & $348 \pm 24$ & $(61.4 \pm 3.2) \times 10^{4}$ \\
4 & 0.6 & $669 \pm 25$ & $>2.63 \times 10^{6}$ \\
8 & 0.2 & $2595 \pm 198$ & $>1.43 \times 10^{6}$ \\
8 & 0.4 & $2803 \pm 377$ & $>3.92 \times 10^{6}$ \\
8 & 0.6 & $2943 \pm 329$ & $>1.88 \times 10^{7}$ \\
& & Superfluid density IACT \\
4 & 0.2 & $98 \pm 5$ & $(17.5 \pm 0.7) \times 10^{3}$ \\
4 & 0.4 & $200 \pm 12$ & $(7.3 \pm 1.4) \times 10^{4}$ \\
4 & 0.6 & $626 \pm 27$ & $>4.89 \times 10^{5}$ \\
8 & 0.2 & $173 \pm 47$ & $>1.59 \times 10^{4}$ \\
8 & 0.4 & $448 \pm 34$ & $>8.36 \times 10^{4}$ \\
8 & 0.6 & $>2340$ & $>3.45 \times 10^{6}$ \\
\hline \hline
\end{tabular}

function of $m$ and reading off the limiting value for large $m$. If convergence to a constant value is not achieved for maximum $m$ used in the measurements, value of $\tau(m)$ for this value is taken to be the lower bound estimate for the true IACT. For a fair comparison we have expressed the IACT in the number of scattering events experienced by the worm, since the simulation time for both cases is directly proportional to the number of these events rather than the number of cycles. Table I shows the results of IACT measurements for the occupation number and superfluid density at a few values of parameters, obtained using both the proposed algorithm and the stochastic series expansion (SSE) heat-bath algorithm.

It can be clearly seen that in all cases, the IACT for the proposed algorithm is much smaller than that for the SSE heat-bath algorithm. As a consequence of a large IACT the heat-bath algorithm starts to develop convergence problems as the average occupation number is increased, making its use impractical in the vicinity of the superfluid transition. On the other hand, the IACT for the proposed algorithm experiences only moderate increase. Moreover, there is no visible slowing down when the system size is doubled even in the vicinity of the critical point, $n_{c} \sim 0.6$. This can be seen by comparing the IACTs for $L=4$ divided by the total number of sites, $L^{3}$, with those for $L=8$. Therefore, the present algorithm is perfectly suitable for simulations near the critical point and also inside the superfluid phase.

In summary, we have described a construction of an efficient QMC algorithm for the simulation of softcore boson models on the lattice, based on the coarse-grained algorithms for the spin models. By establishing the relationship between the boson and spin operators in the infinite $S$ (total spin quantum number) limit, we have mapped the model of noninter- 
acting bosons on a lattice to a spin $X Y$ model in a magnetic field. We have demonstrated that the limit of infinite $S$ may be taken directly in the algorithm, leading to improved performance and absence of systematic errors. The resulting algorithm was found to perform better than existing algorithms. The result of applications of the present algorithm to other models, such as the Bose Hubbard model, will be reported elsewhere [10].
We are grateful to the Swedish National Allocation Committee (SNAC) and National Supercomputer Center in Linköping (Sweden) for providing computer time used for a major part of testing and simulations. The work of N.K. was supported by Grants-in-Aid for Scientific Research Program (Grant No. 14540361) from Monka-sho, Japan. J.S. gratefully acknowledges financial support from the Swedish Foundation for Strategic Research.
[1] S. Sachdev, Quantum Phase Transitions (Cambridge University Press, Cambridge, 1999).

[2] M. Greiner et al., Nature (London) 415, 39 (2002).

[3] V.A. Kashurnikov, N.V. Prokof'ev, and B.V. Svistunov, e-print cond-mat/0202510.

[4] G.G. Batrouni et al., e-print cond-mat/0203082.

[5] K. Harada and N. Kawashima, Phys. Rev. E 66, 056705 (2002)
[6] J. Holstein and N. Primakoff, Phys. Rev. 58, 1098 (1940).

[7] B.B. Beard and U.-J. Wiese, Phys. Rev. Lett. 77, 5130 (1996).

[8] E.L. Pollock and D.M. Ceperley, Phys. Rev. B 36, 8343 (1987).

[9] O.F. Syljuåsen and A.W. Sandvik, Phys. Rev. E 66, 046701 (2002).

[10] J. Šmakov, K. Harada, and N. Kawashima (unpublished). 